\title{
Small-Energy Rotational Transitions in Slow-Neutron Scattering by Water*
}

\author{
SIDNEY YIP† AND R. K. OSBORN \\ Department of Nuclear Engineering, The University of Michigan, Ann Arbor, Michigan
}

(Received 26 April 1963; revised manuscript received 27 May 1963)

\begin{abstract}
A model which treats one rotational degree of freedom as hindered and the other as free and all translational degrees of freedom as hindered has been employed to calculate neutron differential scattering cross section of water in the region of small-energy transfers. The distribution is found to be sensitive to the presence of free-rotation transitions. It is suggested that such transitions present additional complexities in the study of molecular center-of-mass motions from high-resolution scattering data.
\end{abstract}

\section{INTRODUCTION}

$I^{\mathrm{N}}$ $\mathrm{N}$ a previous paper ${ }^{1}$ a model describing hindered rotational motions of polar molecules is used in discussing slow-neutron scattering by condensed systems. The hindrance arises as a result of the coupling between the permanent electric dipole moment of the molecule and an internal field generated by intermolecular interactions. This coupling, however, does not restrict all the rotational degrees of freedom; consequently, the cross section also contains terms corresponding to smallenergy transfers due to excitations of free rotations.

If the model is applied to water, the hindered rotation lines would correspond to the experimentally observed broad band of transitions centered about $\sim 0.06 \mathrm{eV} .^{2}$ Nelkin, ${ }^{3}$ in an earlier work, has treated molecular rotations in water, but in describing the entire rotational motion in terms of a single torsional oscillation his approach does not allow for the possibility of free rota-

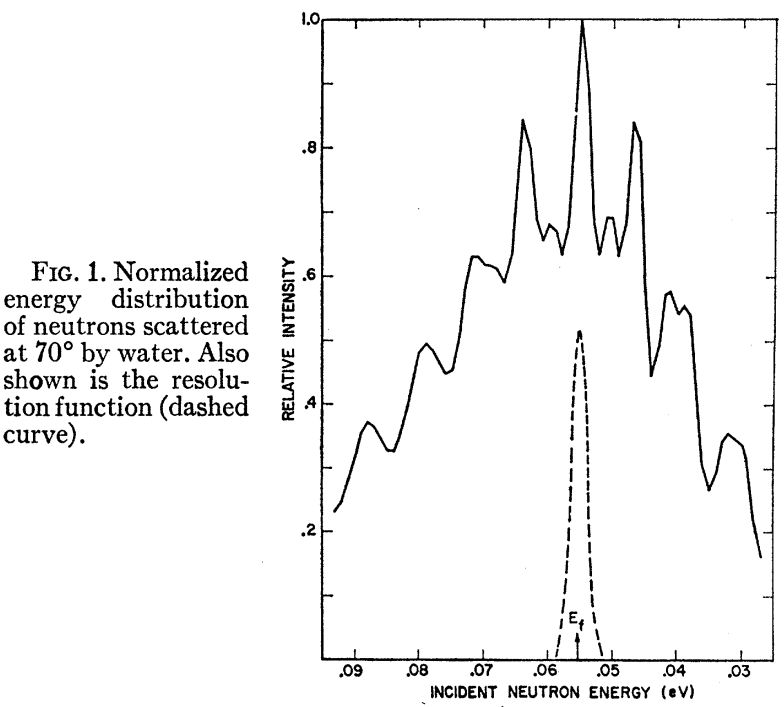

* Supported in part by the National Science Foundation.

$\dagger$ Postdoctoral Fellow, Institute of Science and Technology, The University of Michigan.

${ }^{1}$ S. Yip and R. K. Osborn, Phys. Rev. 130, 1860 (1963). Hereafter this paper is referred to as $\mathrm{I}$.

2 D. J. Hughes, H. Palevsky, W. Kley, and E. Tunkelo, Phys. Rev. 119, 872 (1960); K. Larsson, S. Holmyrd, and K. Otnes, in Proceedings of Symposium on Inelastic Scattering of Neutrons in Solids and Liquids (International Atomic Energy Agency, Vienna, 1961)

${ }^{3}$ M. Nelkin, Phys. Rev. 119, 741 (1960). tions. If, indeed, part of the rotational motions of a water molecule can be regarded as unrestricted it would then be of interest to determine whether such motions can be observed by means of neutron scattering.

In this paper we investigate the effects of free rotation and hindered translation transitions on the scattered neutron energy spectrum in the region around the "quasielastic peak." Numerical results for water, based on the assumption of a simple oscillator description of molecular translations, show that rotational effects will, indeed, influence the observed spectrum in a good resolution experiment. It, thus, appears that one should not, without consideration of the rotational degrees of freedom, attribute any structure or lack of it in the distributions solely to characteristics of center-of-mass motions.

\section{CALCULATION}

The energy and angular differential scattering cross section for a system of molecules is

$$
\begin{aligned}
\sigma\left(E_{i} \rightarrow E_{f}, \theta\right) & =\frac{1}{2 \pi N}\left(\frac{E_{f}}{E_{i}}\right)^{1 / 2} \sum_{l l^{\prime} \alpha \alpha^{\prime}} A_{l \alpha} A_{l^{\prime} \alpha^{\prime}} \\
& \times \int_{-\infty}^{\infty} d t\left\langle\chi_{\alpha \alpha^{\prime}}{ }^{\mathrm{tr}}\right\rangle_{T}\left\langle\chi_{\alpha \alpha^{\prime}}{ }^{\mathrm{rot}}\right\rangle_{T}\left\langle\chi_{\alpha \alpha^{\prime}}{ }^{\mathrm{vib}}\right\rangle_{T} e^{-i \epsilon t},
\end{aligned}
$$

where we use the same notations as I. Here we have assumed that the intermediate scattering function $\left\langle\chi_{\alpha \alpha^{\prime}}\right\rangle_{T}$ can be written as a product of three functions associated with molecular translations and rotations, and internuclear vibrations. An expression for $\left\langle\chi_{\alpha \alpha}{ }^{\text {,ot }}\right\rangle_{T}$ in the case of a symmetric molecule has been derived in I,

$$
\begin{gathered}
\left\langle\chi_{\alpha \alpha^{\prime}}{ }^{\mathrm{rot} t}\right\rangle_{T}=4 \pi \sum_{l k \zeta K M} j_{l}\left(\kappa b_{\alpha}\right) j_{l}\left(\kappa b_{\alpha^{\prime}}\right) Y_{l}{ }^{k *}\left(\hat{b}_{\alpha^{\prime}}\right) Y_{l}{ }^{k}\left(\hat{b}_{\alpha}\right) P_{\zeta K M} \\
\quad \times\left[\exp \left\{i t B k\left[\left(I / I_{z}\right)(2 K-k)+M-K\right]\right\}+W_{1}(t)\right] . \quad(2)
\end{gathered}
$$

The term $W_{1}(t)$ represents the contribution due to hindered rotation excitations. Since it does not directly contribute to small-energy transitions, this term will be neglected in the present calculation.

In order to simplify the computation using Eq. (2) we note that the rotator energy is given by

$$
\begin{aligned}
E_{\zeta K M}=-\lambda+ & (2 \lambda B)^{1 / 2}(2 \zeta+|K-M|+1) \\
& +B K^{2}\left[\left(I / I_{z}\right)-1\right]+B M K \\
& -\frac{1}{4} B[2 \zeta(\zeta+1)+2 \zeta|K-M| \\
& \left.+|K-M|-|K-M|^{2}+2\right]
\end{aligned}
$$


Since $(2 \lambda B)^{1 / 2}$, which is the energy transfer in a hindered rotation excitation, is considerably greater than $B=(2 I)^{-1}$, the terms in Eq. (2) with $K \neq M$ are much more severely attenuated by the Boltzmann factor $P_{\zeta K M}$ relative to the terms with $K=M$. Thus, as a first approximation we consider only the $K=M$ contribution

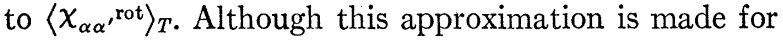
the practical reason of numerical simplification, we see that as a result the cross section depends only upon $I_{z}$, the moment of inertia about the symmetry axis. The approximation therefore allows us to apply Eq. (2) to an asymmetrical molecule without having to specify a value for $I$, which becomes the "effective" moment of inertia for the other two axes.

Equation (1) is now employed for the calculation of the energy distribution of slow neutrons scattered by water. The incoherent cross section, which represents about $95 \%$ of the scattering, is written as

$$
\begin{aligned}
\sigma\left(E_{i} \rightarrow E_{f}, \theta\right)= & \frac{\sigma_{b}}{4 \pi}\left(\frac{E_{f}}{E_{i}}\right)^{1 / 2} \exp \left[-\frac{18 E_{R}}{E_{v}}\right] \exp \left[-\frac{E_{R}}{\omega} \operatorname{coth} \frac{\omega}{2 T}\right] \\
& \times \sum_{n=-\infty}^{\infty} I_{n}\left(\frac{E_{R}}{\omega} \operatorname{csch} \frac{\omega}{2 T}\right) \exp \left[-\frac{n \omega}{2 T}\right] \sum_{l=0}^{\infty}(2 l+1) j_{l}{ }^{2}(\kappa b)\left\{\left[P_{l}{ }^{0}(x)\right]^{2} \delta(\epsilon-n \omega) \sum_{M=-\infty}^{\infty} \exp \left[-\frac{B^{\prime} M^{2}}{T}\right]\right. \\
& \left.+\sum_{k=1}^{l} \frac{(l-k) !}{(l+k) !}\left[P l^{k}(x)\right]^{2} \sum_{M=-\infty}^{\infty}\left(2-\delta_{M 0}\right) \delta\left(\epsilon-n \omega+B^{\prime} k^{2}-2 B^{\prime} M k\right) \exp \left[-B^{\prime} M^{2} / T\right]\right\},
\end{aligned}
$$

where $B^{\prime}=\left(2 I_{z}\right)^{-1}$ is the rotational constant, $E_{R}=\kappa^{2} / 2 M$ the recoil energy, $I_{n}$ the modified Bessel function, $P_{l}{ }^{k}$ the associated Legendre polynomial, and $\gamma=\cos ^{-1} x$ is the angle between the hydrogen atom and the axis of symmetry. In writing Eq. (4) we have assumed that the translational degrees of freedom are hindered and can be approximately characterized by an oscillator model with characteristic frequency $\omega_{0}{ }^{4}$ The effect of zero-point vibration of the lowest internal vibration mode $\left(E_{v}=0.2\right.$ $\mathrm{eV}$ ) is also included. The oscillator description of centerof-mass motions is not unreasonable in view of observations of solid-like behavior of water in slow-neutron scattering studies. ${ }^{2}$ It is admittedly a very crude representation of the physical situation since this model neither admits a spectrum of translational frequencies nor takes into account the fact that the oscillational

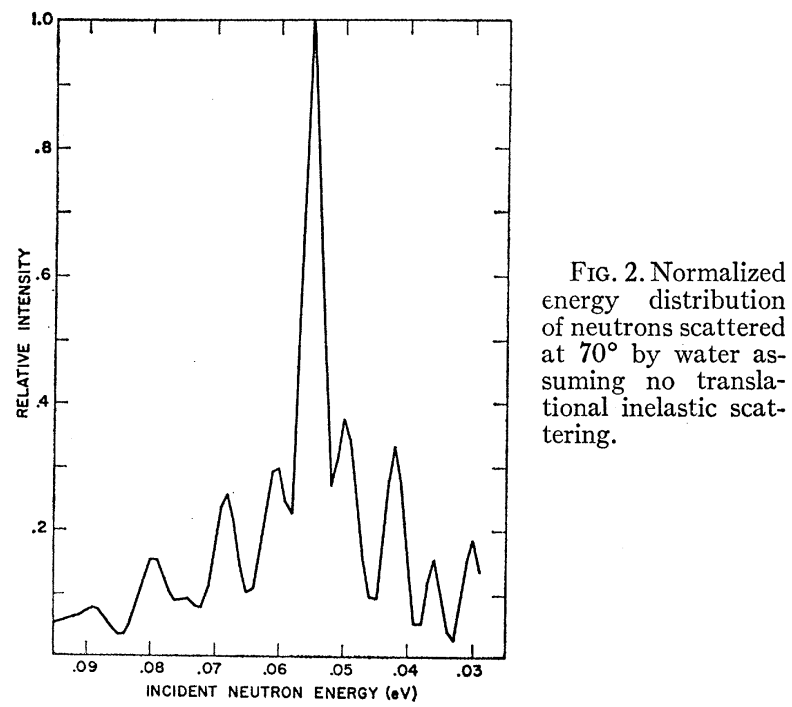

${ }^{4}$ S. Yip, thesis, The University of Michigan, 1962 (unpublished). states have finite lifetimes and are subject to constant fluctuations. For a more elaborate treatment of $\left\langle\chi^{\text {tr }}\right\rangle_{T}$ one may employ a crystalline dislocation model recently proposed by Rosenbaum ${ }^{5}$ or a stochastic model of Rahman, Singwi, and Sjölander. ${ }^{6}$

The contributions from the translational and rotational transitions can be readily exhibited as limiting cases. For $\omega \rightarrow \infty$ only the $n=0$ terms survive and we find

$$
\begin{aligned}
\sigma\left(E_{i}\right. & \left.\rightarrow E_{f}, \theta\right) \\
= & \frac{\sigma_{b}}{4 \pi}\left(\frac{E_{f}}{E_{i}}\right)^{1 / 2} \sum_{l=0}^{\infty}(2 l+1) j_{l}{ }^{2}(k b)\left\{\left[P_{l}{ }^{2}(x)\right]^{2} \delta(\epsilon)\right. \\
& \times \sum_{M=-\infty}^{\infty} e^{-B^{\prime} M^{2} / T}+\sum_{k=1}^{l} \frac{(l-k) !}{(l+k) !}\left[P_{l}^{k}(x)\right]^{2} \\
& \left.\times \sum_{M=-\infty}^{\infty}\left(2-\delta_{M 0}\right) e^{-B^{\prime} M^{2} / T} \delta\left(\epsilon+B^{\prime} k^{2}-2 B^{\prime} M k\right)\right\} .
\end{aligned}
$$

Equation (5) is the cross section appropriate to a rotator whose "apparent" energy is $B^{\prime} M^{2}$ and the selection rules are $\Delta M= \pm s, s$ any integer or zero. The inelastic spectrum, therefore, consists of transitions of $1,3,4,5,7, \cdots$, etc., in units of $B^{\prime}$. On the other hand, for $b \rightarrow 0$ and $I_{z} \rightarrow 0$, only the $l=0$ and $M=0$ terms remain, and we have the well-known cross section of a simple oscillator,

$$
\begin{aligned}
\sigma\left(E_{i}\right. & \left.\rightarrow E_{f}, \theta\right)=\frac{\sigma_{b}}{4 \pi}\left(\frac{E_{f}}{E_{i}}\right)^{1 / 2} \exp \left[-\frac{E_{R}}{\omega} \operatorname{coth} \frac{\omega}{2 T}\right] \\
& \times \sum_{n=-\infty}^{\infty} \delta(\epsilon-n \omega) I_{n}\left(\frac{E_{R}}{\omega} \operatorname{csch} \frac{\omega}{2 T}\right) \exp \left[-\frac{n \omega}{2 T}\right] .
\end{aligned}
$$

${ }^{5}$ M. Rosenbaum, Bull. Am. Phys. Soc. 8, 32 (1963); also, The University of Michigan Tech. Rept. O3712-4-T, 1963 (unpublished).

${ }^{6}$ A. Rahman, K. S. Singwi, and A. Sjölander, Phys. Rev. 126, 997 (1962). 
The resulting spectrum is the familiar series of equally spaced lines.

By means of Eq. (4) we have computed the energy spectrum for $70^{\circ}$ scattering by water at $23^{\circ} \mathrm{C}$. The calculation, using the values $B^{\prime}=1.6 \times 10^{-3} \mathrm{eV}, x=0.61$, and $\omega=8.69 \times 10^{-3} \mathrm{eV}$, is performed to correspond to measurements made with a triple-axis crystal spectrometer in which the incident neutron spectrum is fixed. Total resolution of the experiment is taken to be $5.6 \%$ at $0.055 \mathrm{eV}$. The average of Eq. (4) over both incident and analyzing spectra is given in Fig. 1. Comparison of this result with preliminary measurements ${ }^{7}$ shows qualitative agreement which is encouraging in view of the crudeness of the oscillator description, the fact that the water molecule is asymmetrical, and the $K=M$ approximation.

In order to illustrate the structure of the free-rotation transitions we show in Fig. 2 the same cross section as in Fig. 1 but without any inelastic scattering associated with center-of-mass motions $(n=0)$. The spectrum consists of pronounced energy transfers of $5,13,18,25$, and $34 \mathrm{meV}$. Although the cross section itself is asymmetric with respect to up and down scattering, the apparent preference for the neutron to gain rather than to lose the same amount of energy is due to the fact that we have averaged the cross section over energy-dependent resolution functions. The intensities of the rotational lines are seen to be weaker than those associated with translational transitions. In fact, using an ideal-gas description of translations we find, as shown in Fig. 3, that the rotational structure is completely smeared out out by the broad distribution characteristic of free translations.

The existence of small discrete energy transfers in water was first reported by Hughes and co-workers, ${ }^{2}$ and the same transitions, in a somewhat less pronounced manner, were later also found by Larsson et $a l .^{2}$ On the other hand, Pope and co-workers ${ }^{8}$ reported only a broad distribution and no evidence of discrete transitions. Since it is the part of the spectrum near the quasi-elastic peak that has been used to infer the nature of center-ofmass motions, it is just not clear whether the water molecule is translationally hindered or not. ${ }^{9}$ Moreover,

${ }^{7}$ J. S. King, W. Myers, R. K. Osborn, and S. Yip, Bull. Am. Phys. Soc. 8, 41 (1963).

${ }_{8}$ N. K. Pope, B. N. Brockhouse, R. M. Johnson, and M. Sakamoto, Bull. Am. Phys. Soc. 7, 24 (1962).

${ }^{9}$ P. A. Egelstaff, B. C. Haywood, and I. M. Thorson, in Proceedings of Symposium on Inelastic Scattering of Neutrons in Solids and Liquids (International Atomic Energy Agency, Vienna, 1963).

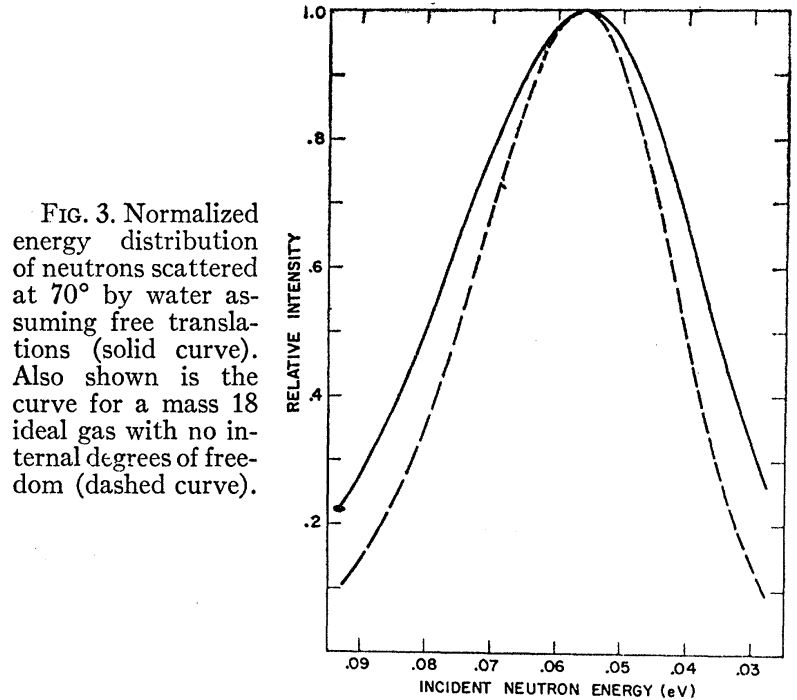

the present considerations show that free rotational effects can also give rise to structure in the same energy region. Thus, at this stage one can only conclude that an interpretation of measurements should take into account this additional degree of freedom.

It is possible, since practically all the experimental data were obtained with cold neutrons, that high-resolution measurements with slow neutrons $\left(E_{i} \sim 10^{-2} \mathrm{eV}\right)$ can be useful in determining the existence of structure in the energy distribution. ${ }^{7}$ In addition to more intense lines as a result of increase in momentum transfer, one also has the advantage of being able to examine both up and down scattering.

The small-energy rotational transitions do not appear in the case of linear molecules. ${ }^{1}$ It is, therefore, suggested that measurements ${ }^{10}$ involving such molecules are better suited for the study of molecular translations. Alternatively, monatomic systems such as liquid lead ${ }^{5,6,11}$ should provide a most direct test of specific models of center-of-mass motions.

\section{ACKNOWLEDGMENTS}

We wish to thank Professor J. S. King for helpful discussions. The computations were performed on the IBM 7090 at The University of Michigan Computing Center.

${ }^{10} \mathrm{H}$. Boutin, G. Safford, and V. Brajovic, Bull. Am. Phys. Soc. 7, $500(1962)$

${ }_{11}$ B. N. Brockhouse and N. K. Pope, Phys. Rev. Letters 3, 259 (1959). 